\title{
Enseñanza-Aprendizaje Transpersonal en el nivel de actividad física del Adulto Mayor
}

Transpersonal teaching-training at the level of physical activity of the elderly Victoria Ramírez-Alcántara $^{a}$, Diana García-Monroy ${ }^{b}$, Viridiana Pedraza-Gonzaga ${ }^{c}$, Gustavo Ortiz-Hernández ${ }^{d}$, Gabriela Maldonado-Muñiz ${ }^{e}$, Claudia A. Trejo-García ${ }^{f}$.

\begin{abstract}
:
Introduction: Watson recognizes that caring is a fundamental part of being and is the most primitive act that a human being performs. Objective: to evaluate the effect of teaching and learning on the level of physical activity of the older adult. Methodology: Using a quantitative, longitudinal and pre-experimental approach to study. The transpersonal teaching and learning intervention was implemented in 3 sessions per week of 50 minutes for 3 months, with 24 participants of 60 years and more. Designing an educational program to increase the level of physical activity based on Watson's theory "Theory of Human Caring", in the proc ess caritas. The level of physical activity was determined through the GPAQ instrument (Global Physical Activity Questionnaire, version 2). Results: $n=24$, the age range was 60 to 90 years, $100 \%$ were women. The level of physical activity in the first measurement was $58.3 \%$ high, $29.2 \%$ moderate and $12.5 \%$ low. After re-evaluating the teaching-learning effect of physical activity, favorable results were obtained at a level of $100 \%$ in all participants. Conclusion: Teaching transpersonal learning increases the level of physical activity of the elderly.
\end{abstract}

Keywords:

Nursing Care, Face Process, Nursing Intervention, Teaching, Learning, Elderly

\section{Resumen:}

Introducción: Watson reconoce que el cuidar es parte fundamental del ser y es el acto más primitivo que un ser humano realiza. Objetivo: evaluar el efecto de enseñanza aprendizaje transpersonal en el nivel de actividad física del adulto mayor. Metodología: Utilizando un método de estudio con enfoque cuantitativo, longitudinal y pre-experimental. La intervención de enseñanza aprendizaje se implementó en 3 sesiones por semana de 50 minutos por 3 meses, se contó con 24 participantes de 60 años y más. Diseñando un programa educativo para aumentar el nivel de actividad física con base a la teoría de Watson titulado "Teoría del cuidado humanizado", en el proceso caritas. Se determinó el nivel de actividad física a través del instrumento GPAQ (Global Physical Activity Questionnaire, versión 2). Resultados: $n=24$, el rango de edad fue de 60 a 90 años, el $100 \%$ eran mujeres. El nivel de actividad física la primera medición se obtuvo $58.3 \%$ nivel alto, $29.2 \%$ moderado y $12.5 \%$ bajo. Después de reevaluar el efecto enseñanza- aprendizaje de la actividad física se obtuvieron resultados favorables en un nivel del 100\% en todos los participantes. Conclusión: La enseñanza aprendizaje transpersonal aumenta el nivel de actividad física del adulto mayor.

\section{Palabras Clave:}

Cuidado de enfermería, Proceso caritas, Intervención de enfermería, Enseñanza, Aprendizaje, Adulto mayor

Universidad Autónoma del Estado de Hidalgo, Escuela Superior de Tlahuelilpan, Alumno de la Licenciatura en Enfermería, ORCID: https//orcid.org/0000-0002-1803-0369, Email: ramirezalcantaravictoria.4c@ gmail.com

b Universidad Autónoma del Estado de Hidalgo, Escuela Superior de Tlahuelilpan, Alumno de la Licenciatura en Enfermería, ORCID: https//orcid.org/0000-0002-5816-0678, Email: garciamonroydiana@ gmail.com

Universidad Autónoma del Estado de Hidalgo, Escuela Superior de Tlahuelilpan, Alumno de la Licenciatura en Enfermería, ORCID: https//orcid.org/0000-0002-2892-2264, Email: virigonzaga29@gmail.com

d Universidad Autónoma del Estado de Hidalgo, Escuela Superior de Tlahuelilpan, Alumno de la Licenciatura en Enfermería, ORCID: https//orcid.org/0000-0002-0894-5645, Email: gusssooo_hotmail.com

Autor de Correspondencia, Universidad Autónoma del Estado de Hidalgo, Escuela Superior de Tlahuelilpan, ORCID: https://orcid.org/0000-0002-4967-1812,Email: gmaldonado@uaeh.edu.mx

f Universidad Autónoma del Estado de Hidalgo, Escuela Superior de Tlahuelilpan, ORCID: https://orcid.org/0000-0003-2205-7879, Email: ctrejo@uaeh.edu.mx

Fecha de recepción: 07/05/2020, Fecha de aceptación: 17/05/2020, Fecha de publicación: 05/07/2020 


\section{Introducción}

Los adultos mayores sufren múltiples cambios en la composición corporal. La pérdida progresiva de musculo esquelético y su fuerza forma parte del envejecimiento. A este cambio se le ha atribuido, al menos en parte, la disminución de la funcionalidad motriz y a todo su estilo de vida, repercutiendo en su capacidad funcional de la población geriatra, afectando gravemente su calidad de vida (Landinez Parra, Contreras Valencia, and Castro Villamil 2012).

Los 10 procesos de Caritas de Watson de la teoría del cuidado transpersonal:

1. Mantener los valores humanistas-altruistas a través de la práctica de la bondad amorosa, la compasión y la ecuanimidad con uno mismo y con los demás.

2. Estar auténticamente presente, habilitando el sistema de fe/esperanza/creencia; honrando el interior subjetivo, el mundo de vida de uno mismo y de los demás.

3. Ser sensible a sí mismo y a los demás cultivando sus propias prácticas espirituales; yendo más allá del ego para presencia transpersonal.

4. Desarrollar y mantener una relación de amor y confianza.

5. Permitir la expresión de sentimientos positivos y negativos; escuchar auténticamente al otro la historia de la persona.

6. Creativamente solución de problemas/ "búsqueda de soluciones" a través de un proceso de cuidado; uso completo de sí mismo y el arte de las prácticas de cuidado y curación mediante el uso de todas las formas de conocimiento.

7. Promoción de la enseñanza y el aprendizaje transpersonal dentro del contexto de las relaciones de cuidado; permanecer dentro del marco de referencia de otros.

8. Crear un entorno de curación a todos los niveles, en el que la auténtica presencia de cuidado potencie la integridad, la belleza, la comodidad, la dignidad y la paz.

9. Asistir reverentemente con las necesidades básicas como actos sagrados, tocando la mente-cuerpo-espíntu de otros, que sostiene la dignidad humana.

10. Abrirse a lo espiritual, a los misterios, a las incógnitas; permitir los milagros (Wagner, Watson, and Cara 2020).

La promoción de la enseñanza-aprendizaje interpersonal deriva a: "Participar de una verdadera enseñanzaaprendizaje que atienda a la unidad del sery de su sentido y que trate de mantenerse en el marco referencial del otro". Este factor de cuidado es el que más ayuda al usuario a ser partícipe activo del estado de su propia salud y a tomar las decisiones a favor de mantenerla, recuperarla, mejorarla, o en otros casos a prepararse para determinados eventos (Urra M, Jana A, and García V 2011).

\section{Planteamiento del problema o exposición del caso}

La actividad física recomendada por la Organización Mundial de la Salud (OMS) en los adultos mayores consiste en actividades recreativas 0 de ocio, desplazamientos (por ejemplo, paseos caminando o en bicicleta), actividades ocupacionales (cuando la persona todavía desempeña actividad laboral), tareas domésticas, juegos, deportes o ejercicios programados en el contexto de las actividades diarias, familiares y comunitarias. Con el fin de mejorar las funciones cardiorrespiratorias y musculares y la salud ósea y funcional, y de reducir el riesgo de enfermedades no transmisibles, depresión y deterioro cognitivo (OMS 2020).

\section{Conocimiento previo}

No se cuenta con estudios previos donde se utilice la medición del nivel de actividad física con un in strumento recomendado por la OMS y se haga una intervención con base en una teoría de enfermería, dirigida a los adultos mayores, por lo que el objetivo de la investigación fue: Evaluar el efecto de la promoción de la enseñanzaaprendizaje interpersonal en el nivel de actividad física del adulto mayor de la casa del adulto mayor del municipio de Tetepango Hidalgo.

\section{Metodología de diseño o de estudio}

Estudio cuantitativo, longitudinal y pre-experimental. El universo para realizar el trabajo de investigación estuvo conformado por 24 adultos mayores de la Casa del adulto mayor de Tetepango. Se incluyó a personas de 60 años de edad y más, con habilidades motoras tanto finas como gruesas y disponibilidad de tiempo.

\section{V.Aplicación}

En el programa que se ha establecido y de acuerdo a criterios de la Organización Mundial de la Salud, 3 veces a la semana impartiendo en cada día 50 minutos de actividad física, repartiendo los 50 minutos en 3 actividades dentro del mismo lapso de tiempo (Organización Mundial de la Salud (OMS) 2010), realizando ejercicios tanto aeróbicos, fuerza, equilibrio y de flexibilidad (Sociedad Española de Geriatría y Gerontología 2012). Al igual que tomando en cuenta que el proceso enseñanza-aprendizaje transpersonal, la educación como un proceso consciente y organizado de apropiación de los contenidos y las formas de conocer, hacer, vivir y ser, construidos en la experiencia como resultado de la actividad del individuo y su interacción con la sociedad en su conjunto, en el cual se producen 
cambios que le permiten adaptarse a la realidad, transformarla y crecer personalmente (Urra M et al. 2011). Se determinó Nivel de Actividad Física a través del GPAQ (Global Physical Activity Questionnaire). Este instrumento consta de 16 ítems evalúa la actividad física desarrollada en tres dominios (actividad física ocupacional, relacionada con el transporte y actividad física en el tiempo libre) y abarca varios componentes de la actividad física, tales como intensidad, duración y frecuencia, clasificando los resultados en alto, medio y bajo. Tiene una confiabilidad prueba-reprueba con un $\mathrm{CCl}$ (Coeficiente Relación Intra Clase) superiores a 0.73-0.82 post prueba (Angarita Fonseca, Camargo Lemos, and Orórtegui Arenas 2010). Para el análisis de los datos de la investigación se utilizó el paquete estadístico SPSS (Statistical Package for the Social Sciences versión 21 se aplicó tanto descriptivo como inferencial. Los sujetos fueron informados verbalmente y por escrito de los objetivos y metodología del trabajo, con especial consideración en los riesgos y posibles molestias que pudiera ocasionar el estudio. El presente trabajo de investigación se desarrolló respetando la Carta de Helsinki para investigaciones en seres humanos y se solicitó a todos los participantes el consentimiento informado (World Medical Association 2013).

\section{Resultados}

La muestra estuvo conformada por 24 personas de género femenino, con un rango de edad de 60 a 90 años.

En la primera medición del nivel de actividad física se obtuvo 14 en nivel alto, 7 en nivel moderado, y 3 en nivel bajo de acuerdo a la clasificación del GPAQ. Después de realizar las intervenciones basadas en el efecto enseñanza- aprendizaje se evaluó el nivel de actividad física alcanzando un nivel del $100 \%$ en nivel alto todas las participantes. Ver tabla No. 1

Tabla No.1 Frecuencia y porcentaje de nivel de actividad física de los adultos mayores de la casa del adulto mayor del municipio de Tetepango, Hidalgo, México, 2019

\begin{tabular}{lll}
\hline Nivel de & Primera & Segunda \\
actividad física & Medición & Medición \\
Alto. & $14(58.3 \%)$ & $24(100 \%)$ \\
Medio. & $7(29.2 \%)$ & \\
Bajo. & $3(12.5 \%)$ & \\
\hline Fuente: GPAQ & & $\mathrm{n}=24$
\end{tabular}

\section{Discusión}

En el estudio "actividad física y calidad de vida de la población adulta mayoren España”, por Rubio Castañeda y cols., obtuvieron un $18.7 \%$ en nivel alto, $74.8 \%$ en nivel moderado y $6.5 \%$ en nivel bajo ya que su población solo realizaba actividad física en sus tiempos libres y su población no estaban integrados a ninguna institución (Rubio Castañeda, Tomás Aznar, and Muro Baquero
2017), mientras que en los adultos mayores de Tetepango se obtuvo un $58.3 \%$ en el nivel alto, $29.2 \%$ en nivel medio y $12.5 \%$ en nivel bajo de actividad física ya que participan en un programa para adultos mayores, por lo que realizar algún tipo de actividad física es parte de su vida diaria y no solo cuando estos tienen un tiempo libre.

\section{Conclusiones}

El nivel de actividad física del adulto mayor aumenta con intervención de enfermería la enseñanza aprendizaje interpersonal, con base el proceso Caritas de la teoría del Cuidado Transpersonal de Jean Watson.

\section{Agradecimientos}

A los adultos mayores participantes.

\section{Referencias}

Angarita Fonseca, Adriana, Diana Marina Camargo Lemos, and Myriam Orórtegui Arenas. 2010. "Reproducibilidad Del Tiempo En Posición Sedente Evaluado Con El International Physical Activity Questionnaire (IPAQ) y El Global Physical Activity Questionnaire ( GPAQ )." Med UNAB 13(1):5-12.

Landinez Parra, Nancy Stella, Katherine Contreras Valencia, and Ángel Castro Villamil. 2012. "Proceso de Envejecimiento, Ejercicio y Fisioterapia.” Revista Cubana de Salud Pública 38(4):562-80.

OMS. 2020. "La Actividad Física En Los Adultos Mayores." Estrategia Mundial Sobre Régimen Alimentario, Actividad Física y Salud 3 pantallas. Retrieved May 7, 2020 (https://www.who.int/dietphysicalactivity/factsheet_olderad ults/es/).

Organización Mundial de la Salud (OMS). 2010. Recomendaciones Mundiales Sobre Actividad Física Para La Saldud. Suiza: Organización Mundial de la Salud.

Rubio Castañeda, Francisco Javier, Concepción Tomás Aznar, and Carmen Muro Baquero. 2017. "Medición de La Actividad Física En Personas Mayores de 65 Años Mediante El IPAQ-E: Validez de Contenido, Fiabilidad y Factores Asociados." Rev Esp Salud Pública 91(e201701004):1-12.

Sociedad Española de Geriatría y Gerontología. 2012. "Guía de Físico Para Mayores.” Tu Salud En Marcha, 10-55.

Urra M, Eugenia, Alejandra Jana A, and Marcela García V. 2011. "Algunos Aspectos Esenciales Del Pensamiento de Jean Watson y Su Teoría de Cuidados Transpersonales." Ciencia y Enfermería 17(3):11-22.

Wagner, A. Lynne, Jean Watson, and Chantal Cara. 2020. "Core Concepts of Jean Watson's Theory of Human Caring ${ }^{\circledR} /$ Unitary Caring Science ${ }^{\circledR}$." Watson Caring Sciencie Institute 1-8. Retrieved May 7, 2020 (https://www.watsoncaringscience.org/files/PDF/watsonstheory-of-human-caring-core-concepts-and-evolution-tocaritas-processes-handout.pdf).

World Medical Association. 2013. "World Medical Association Declaration of Helsinki. Ethical Principles for Medical Research Involving Human Subjets." JAMA 310 (20):219194. 